\title{
Pretrial functional connectivity differentiates behavioral outcomes during trace eyeblink conditioning in the rabbit
}

\author{
Matthew P. Schroeder, ${ }_{1}^{1}$ Craig Weiss, ${ }^{1}$ Daniel Procissi, ${ }^{2}$ Lei Wang, ${ }^{2,3,4}$ \\ and John F. Disterhoft ${ }^{1,4}$ \\ ${ }^{1}$ Department of Physiology, Feinberg School of Medicine; ${ }^{2}$ Department of Radiology, Feinberg School of Medicine; ${ }^{3}$ Department \\ of Psychiatry and Behavioral Sciences, Feinberg School of Medicine, Northwestern University, Chicago, Illinois 60611, USA
}

\begin{abstract}
Fluctuations in neural activity can produce states that facilitate and accelerate task-related performance. Acquisition of trace eyeblink conditioning ( $\mathrm{tEBC}$ ) in the rabbit is enhanced when trials are contingent on optimal pretrial activity in the hippocampus. Other regions which are essential for whisker-signaled tEBC, such as the cerebellar interpositus nucleus (IPN), somatosensory and prelimbic cortices, may also show optimal connectivity prior to successful performance. Functional magnetic resonance imaging ( $\mathrm{FMRI}$ ) was acquired in nine rabbits during $\mathrm{tEBC}$ on the first and tenth days of initial training and once again after a 30-d, training-free hiatus. Data acquired during the intertrial interval was parsed depending on whether or not a conditioned response (CR) occurred on the upcoming trial and seed-based functional connectivity was calculated among the IPN, hippocampus, somatosensory, and prelimbic cortices. Functional connectivity between the left somatosensory cortex and right IPN, regions critical for establishing and producing CRs evoked by right vibrissae vibration and right corneal airpuff, was significantly negative prior to successful, CR trials as compared with unsuccessful, non-CR trials. Differences were not observed for any of the other possible combinations of connectivity. Our results demonstrate that specific pretrial functional connectivity exists within the rabbit brain and differentiates between upcoming behavioral response outcomes. Online analysis of network fluctuations has the potential to be used as the basis for therapeutic interventions to facilitate learning and memory.
\end{abstract}

[Supplemental material is available for this article.]

Trace eyeblink conditioning (tEBC) is a learning paradigm that shows accelerated behavioral acquisition when trial presentation is contingent on the state of theta power in the hippocampus (Seager et al. 2002; Griffin et al. 2004; Hoffmann and Berry 2009; Darling et al. 2011). tEBC consists of a neutral conditioned stimulus (CS) followed by a stimulus-free trace interval and a salient unconditioned stimulus (US; e.g., corneal airpuff) resulting in a conditioned response (CR; extension of the nictitating membrane). The hippocampus (Solomon et al. 1986; Moyer et al. 1990; Kim et al. 1995; Weiss et al. 1999), medial prefrontal cortex (Kronforst-Collins and Disterhoft 1998; Weible et al. 2000; McLaughlin et al. 2002; Takehara et al. 2003), and somatosensory cortex (Galvez et al. 2007) are essential in whiskersignaled trace conditioning. In addition, the neural pathway of the CS, US, and conditioned blink all incorporate the cerebellar interpositus nucleus (IPN) (Woodruff-Pak et al. 1985; Green and Arenos 2007; Plakke et al. 2007; Pakaprot et al. 2009; Hu et al. 2010, 2012; Wu et al. 2012) and it is in this region where information about the CS and US both converge (McCormick et al. 1982; Clark et al. 1984; Yeo et al. 1985; Lavond and Steinmetz 1989; Krupa et al. 1993, 1996; Krupa and Thompson 1995, 1997; Christian and Thompson 2003, 2005).

Learning rates on $\mathrm{EEBC}$ are strongly correlated with the relative magnitude of theta oscillations in the CA1 region of the dorsal hippocampus prior to training trials (Berry and Thompson

\footnotetext{
${ }^{4}$ These authors contributed equally to this work.
}

Corresponding author: jdisterhoft@northwestern.edu

Article is online at http://www.learnmem.org/cgi/doi/10.1101/lm.040220.115.
1978). Previous studies have shown that rabbits receiving trials contingent on high theta power within the CA1 region of the dorsal hippocampus acquired the task significantly faster compared with rabbits receiving trials contingent on low/absent theta power (Griffin et al. 2004; Darling et al. 2011; Seager et al. 2002; Hoffmann and Berry 2009). Moreover, when tEBC trials were presented in the presence of high hippocampal theta power, cerebellar IPN, and hemispheric lobule VI (HVI) local field potentials became time-locked and synchronized in the theta frequency during the trace and post-US period (Hoffmann and Berry 2009).

Altered activity, connectivity or oscillatory states occur in neural circuits prior to successful memory encoding and sensory discrimination (see Cohen et al. 2015, for comprehensive overview). For instance, multiunit neuronal responses in rabbit somatosensory cortex were found to differentiate CR and non-CR trials during the trial period of delay eyeblink conditioning using an auditory CS (Wikgren et al. 2003). Information is encoded in the synchronized firing times of distributed neurons (König et al. 1995; Schneider et al. 2006; Nikolić 2007) and coordinated, interregional oscillations occurring prior to stimulus presentation may produce an optimal brain state to facilitate performance and improve learning and retention. Because of the crucial importance

(C) 2016 Schroeder et al. This article is distributed exclusively by Cold Spring Harbor Laboratory Press for the first 12 months after the full-issue publication date (see http://learnmem.cshlp.org/site/misc/terms.xhtml). After 12 months, it is available under a Creative Commons License (AttributionNonCommercial 4.0 International), as described at http://creativecommons. org/licenses/by-nc/4.0/. 


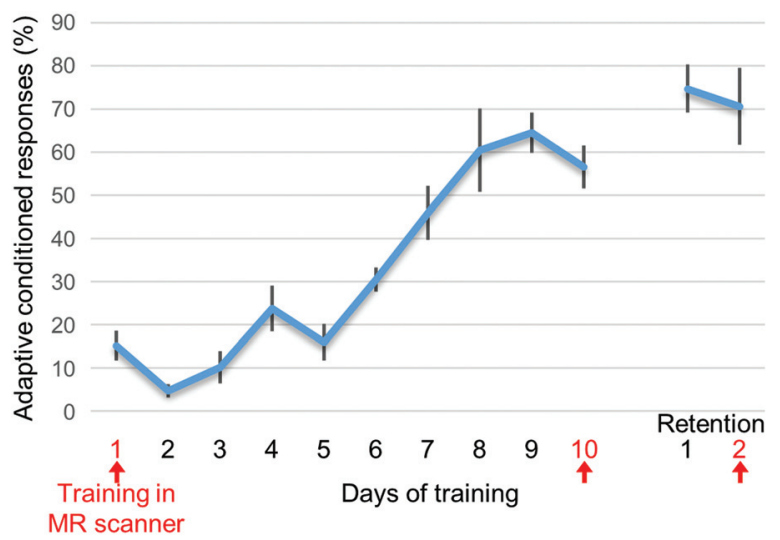

Figure 1. $t E B C$ behavioral results. Rabbits were trained for 10 consecutive days and, in order to test memory retention, two additional days after a 30-d period of no training. Training was performed inside the MR scanner on days 1, 10, and the second day of retention testing (as denoted by the red arrows). Rabbits acquired the tEBC task (mean percent adaptive CRs $\pm S E$ ) over the course of $10 \mathrm{~d}$ and retained the task when tested $30 \mathrm{~d}$ later.

of the IPN, hippocampus, prelimbic, and somatosensory cortex to integrate sensory information and produce behavioral output (see Christian and Thompson 2003; Woodruff-Pak and Disterhoft 2008 and Fig. 1 in Medina et al. 2002 for a comprehensive overview of each pathway), coordinated fluctuations distributed across these brain regions may facilitate learning of tEBC. In this study, we performed tEBC experiments on rabbits while collecting functional magnetic resonance imaging (fMRI) data at three different periods of the learning process (early and late in acquisition, and during retention testing). We hypothesized that, prior to $\mathrm{tEBC}$ trials, functional connectivity among the hippocampus, IPN, somatosensory, and prelimbic cortices would be different depending on the performance of the upcoming trial.

\section{Results}

\section{Behavioral results}

Rabbits reliably learned the tEBC task over $10 \mathrm{~d}$ (Fig. 1). A significant increase in the percentage of adaptive CRs across sessions was revealed (repeated-measures ANOVA, main effect time:
$\left.F_{(11,88)}=21.59, P<0.0001\right)$. The increase in adaptive CRs was maintained during retention testing $30 \mathrm{~d}$ later where adaptive CRs remained high $(\sim 73 \%)$ demonstrating that rabbits retained the learning established during the initial $10 \mathrm{~d}$ of training. On average, rabbits reached behavioral criterion (operationalized as eight adaptive CRs within a 10 trial moving window) by the seventh day.

\section{Pretrial connectivity results}

Functional connectivity during the intertrial interval was significantly different prior to CR and non-CR trials between the left somatosensory cortex (SI) and right cerebellar interpositus nucleus (IPN) (Fig. 2). Prior to CR trials, Z-transformed left SI $\leftrightarrow$ right IPN connectivity was significantly negative compared with non-CR trials across the three scanning days $(-0.101$ versus 0.042 , respectively; average paired difference $=-0.143, F_{(1,8)}=$ $25.2 ; P<0.001$, Fig. 2B). Left SI $\leftrightarrow$ right IPN connectivity prior to CR trials did not significantly differ across the three scanning days. The significant negative connectivity values indicate that signal changes in the left SI during the intertrial interval prior to CR trials is accompanied by signal changes in the right IPN with opposite direction (i.e., signal increase in left SI accompanied by signal decrease in right IPN and vice versa across time). Had they occurred, positive connectivity values would have indicated that signal changes in the left SI during the intertrial interval prior to CR trials is accompanied by signal changes in the right IPN with similar direction (i.e., signal increase in left SI accompanied by signal increase in right IPN and vice versa across time).

To demonstrate the anatomical specificity of the left SI $\leftrightarrow$ right IPN circuit, the Z-transformed connectivity strength of the opposite contralateral (i.e., right SI $\leftrightarrow$ left IPN) and the two ipsilateral comparisons (i.e., left SI $\leftrightarrow$ left IPN, right SI $\leftrightarrow$ right IPN) were examined. Z-transformed connectivity strength prior to CR and non-CR trials was not significantly different for any of the three comparisons, nor for any post hoc comparisons on individual days (Supplemental Fig. 1). Also, no significant main effect for trial type was found among any of the other regional combinations including the hippocampus or prelimbic cortex (Supplemental Figs. 2-5). Post hoc analyses examining the difference between trial types on individual days revealed that six comparisons were significant on an individual day (Supplemental Figs. 2F, 3D,F, 4F, 5B,F). However, we believe these to be false-positives. Eighty-four $t$-tests were performed (three sessions for each of the 28 regional comparisons) and with a $P$-value of 0.05 , it is likely
A

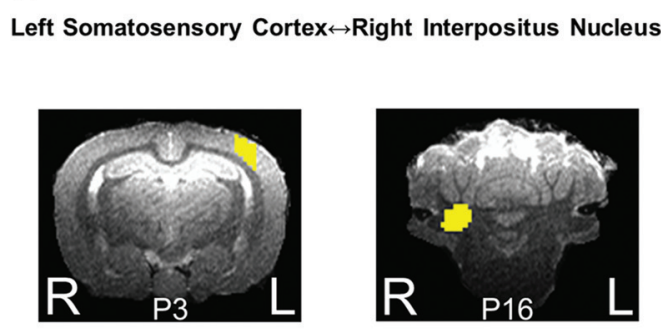

B

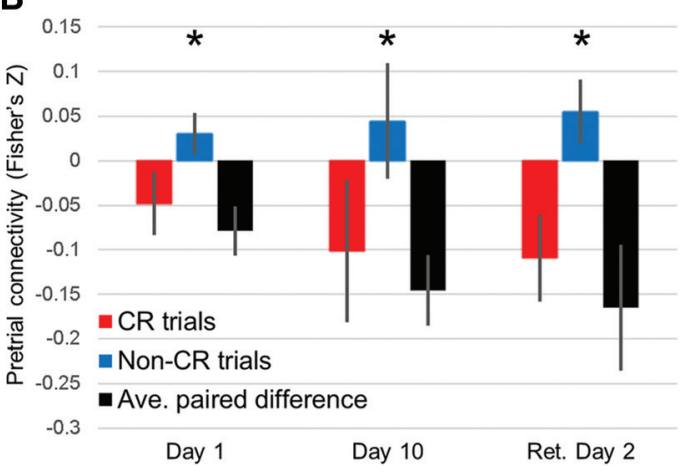

Figure 2. Pretrial connectivity differences between the left somatosensory cortex and right interpositus nucleus are associated with performance of the upcoming trial. (A) Regions of interest for the left somatosensory cortex and right interpositus nucleus. (B) Significant difference in Z-transformed connectivity strength between upcoming $C R$ and upcoming non-CR trials across the $3 \mathrm{~d}$ (days 1,10 , and Retention day 2 ). Bars denote connectivity prior to $\mathrm{CR}$ trials (red), non-CR trials (blue), and the paired difference between the two (black). Asterisks denote significant difference between pretrial connectivity on CR and non-CR trials. See Discussion for explanation of negative connectivity prior to CR trials. 
that $\sim 4$ comparisons would be false-positives. We found six and unlike the left SI $\leftrightarrow$ right IPN comparison, none of the other comparisons show a significant main effect across all three sessions. The six comparisons with $P$ values $<0.05$ are about the number of significant comparisons that we would expect by chance.

Post hoc analyses were conducted to determine whether left SI $\leftrightarrow$ right IPN connectivity strength was correlated with the following aspects of the motor response; mean amplitude of response, duration of response, area under the curve, onset latency of response, peak amplitude of the response, latency to the peak of the response, standard deviation, slope, and rise time of the response (i.e., time to get from $20 \%$ to $80 \%$ of maximum peak) at six distinct time points; baseline period (i.e., $250 \mathrm{msec}$ prior to CS onset), 20 msec after CS onset, the entire period between CS and US onset as well as 200 and $20 \mathrm{msec}$ prior to US onset, and the UR period. The conditioned blink response of a rabbit typically occurs during the $200 \mathrm{msec}$ time period prior to the onset of the airpuff stimulus (UR). We observed a significant positive correlation between the strength of the left $\mathrm{SI} \leftrightarrow$ right IPN connectivity and the duration of the response occurring 200 msec prior to US onset $(r=0.737, P<0.002$; Supplemental Fig. $6 \mathrm{~A}$ ) as well as a significant negative correlation between the strength of the left SI $\leftrightarrow$ right IPN connectivity and the onset of the response occurring $200 \mathrm{msec}$ prior to US onset $(r=-0.717$, $P<0.003$; Supplemental Fig. 6B).

The significant pretrial connectivity difference observed between the left SI $\leftrightarrow$ right IPN could actually be negatively synchronized fluctuations as a result of post-trial encoding if consecutive trials were consistently different types (i.e., non-CR trial followed by a CR trial and vice versa). However, the proportion of consecutive trials of different trial types was $\sim 10 \%$ for each day (Supplemental Fig. 7A). Post-trial connectivity between the left SI and right IPN was examined to determine whether there was differential connectivity after CR and non-CR trials. No significant effect was found across the three scanning days and post-trial connectivity for CR and non-CR trials was not significantly different from zero (Supplemental Fig. 7B). Also, no significant differences were found among any of the other regional combinations including the hippocampus or prelimbic cortex for post-trial connectivity.

\section{Time delay analysis}

A time delay analysis was performed to determine whether connectivity between the left SI and right IPN would become more robust. Lagging the time series of either region relative to the other by one TR (i.e., $2.5 \mathrm{sec}$ ) reduced the left SI $\leftrightarrow$ right IPN connectivity prior to CR and non-CR trials and the paired difference (Fig. 3). Yet, both lags still showed a significant main effect for upcoming trial type (right IPN lagged 1TR: $F_{(1,8)}=8.591 ; P<0.012$; left SI lagged 1TR: $\left(F_{(1,8)}=7.705 ; P<0.025\right)$. When lagging by 2 or 3 TRs (i.e., 5 or $7.5 \mathrm{sec}$ ), no significant differences existed between upcoming CR and non-CR trials and the connectivity for both CR or non-CR upcoming trials approached zero (Fig. 3).

\section{Discussion}

In this study, functional connectivity between the left SI $\leftrightarrow$ right IPN was significantly different depending on upcoming behavioral performance on tEBC trials. The left SI and right IPN, regions critical for tEBC acquisition and memory retrieval (Woodruff-Pak et al. 1985; Galvez et al. 2007) when right whisker vibration is repeatedly paired with an airpuff to the right cornea, showed significant negative connectivity prior to successful CR trials and no significant connectivity prior to unsuccessful non-CR trials. Because of this, the left SI $\leftrightarrow$ right IPN connectivity

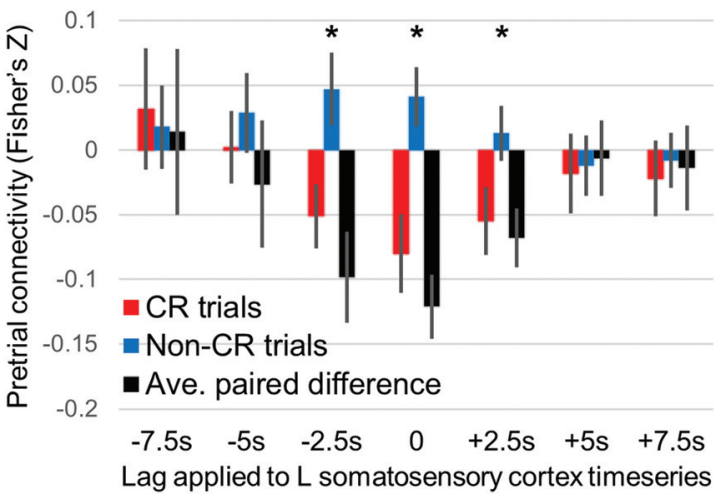

Figure 3. Time delay analysis of left somatosensory cortex and right interpositus nucleus (IPN). Lagging the time series of the left somatosensory cortex by $\pm 2.5 \mathrm{sec}$ (i.e., 1TR) produced significant pretrial connectivity differences between $C R$ and non-CR trials similar to no lag but did not improve the difference. Bars denote connectivity prior to CR trials (red), non-CR trials (blue), and the average paired difference between the two (black).

prior to CR trials demonstrates negative synchrony whereas connectivity prior to non-CR trials is dysynchronous. The laterality of the connection is anatomically correct given that whisker vibrations (CS) and corneal airpuffs (US) were delivered to the right side and the right SI and left IPN did not show the same effect. The left SI $\leftrightarrow$ right IPN connectivity difference prior to CR and non-CR trials is robust and stable even from the first day of tEBC. Fewer CRs were produced early in training potentially because left SI $\leftrightarrow$ right IPN connectivity was not in an optimal pretrial state as often. But as the tEBC task was acquired and retained, the proportion of time that the left SI $\leftrightarrow$ right IPN pretrial connectivity was negatively synchronized in an optimal state increased thereby supporting a greater number of CRs.

The results we report here represent pretrial functional connectivity between the whisker-related cortical region and the cerebellar output that controls the conditioned blink. Prior to non-CR trials, no correlation was observed between the left SI and right IPN whereas negative correlations were seen prior to CR trials. To understand the direction of the connectivity, it is important to understand the anatomical pathways of tEBC. The CS pathway begins with sensory systems sending projections to the ipsilateral pontine nuclei (Glickstein et al. 1980; Brodal 1981; Schmahmann and Pandya 1989, 1991, 1993) followed by mossy fiber axons which decussate across the midline to carry the excitatory CS-related information to the contralateral IPN as well as cerebellar granule cells and eventually Purkinje cells which send inhibitory input to the IPN (for review, see Medina et al. 2002). The connectivity seen between the left SI and right IPN prior to CR trials may result from anticipation for upcoming events during the intertrial interval. Such anticipation can trigger top-down or bottom-up attentional processes (Driver and Frith 2000) which increases the neural gain and coordinates temporally overlapping neuronal processes (Ma et al. 2006; van den Berg et al. 2012) leading to strengthened memory encoding (Miller and Cohen 2001; Chun and Turk-Browne 2007; Noudoost et al. 2010). tEBC trials resulting in a non-CR may be characterized by noisy and inefficient pretrial output from the SI to the IPN and Purkinje cells leading to a noncorrelated state. On the other hand, upcoming trials producing a CR might be characterized by efficient somatosensory cortical excitatory projections to the cerebellar circuit during the intertrial interval. We have previously shown a conditioningspecific decrease in baseline single-neuron activity in primary SI of rabbits during the sessions immediately before and after 
reaching behavioral criterion for whisker-signaled tEBC (Ward et al. 2012) and in delay conditioning with a visual CS, the ipsilateral IPN has a sustained blood-oxygen-level dependent (BOLD) signal increase into the intertrial interval in proportion to the percentage of CR trials within a session (Miller et al. 2003). A progressive decrease in SI baseline activity may result in increased signal-to-noise from the SI prior to CR trials, leading to Purkinje cell delays of IPN activity relative to SI, and/or an increased excitability of cerebellar nuclear neurons projecting to motor neurons controlling the conditioned blink. Either of these possibilities may result in a negative correlation between the left SI and right IPN prior to CR trials. The IPN, being close to the motor output of the conditioned reflex, would then be more excitable in response to cortical input through the mossy fiber collaterals after CS presentation thereby increasing the likelihood of a CR being produced on that trial.

When right whisker vibration is repeatedly paired with an airpuff to the right cornea in trace eyeblink conditioning (tEBC), the left SI and right IPN are important for acquisition and memory retrieval (Woodruff-Pak et al. 1985; Galvez et al. 2007; Green and Arenos 2007; Plakke et al. 2007; Pakaprot et al. 2009; Hu et al. 2010, 2012; Wu et al. 2012). If either are lesioned prior to the start of training, the tEBC task will not be acquired. The necessity of these regions is therefore different than that of the prelimbic cortex. We did not observe a main effect difference for prelimbic or hippocampal connectivity prior to CR and non-CR trials that was consistent across all three sessions. Although significant post hoc differences were observed on individual sessions (Supplemental Figs. 2F, 3D,F, 4F, 5F), we believe these differences to be false-positives. Three of the significant post hoc analyses show a significant difference between the right IPN and right hippocampus on day 1 . The role of the prelimbic cortex is most robust once learning has been established (Takehara et al. 2003; Hattori et al. 2014) therefore our post hoc result is counter to what we would expect. Additionally, the left and right hippocampus shows a significant difference with the right IPN on day 10 only. Permanent lesion studies indicate that the hippocampus is essential for the acquisition of tEBC (Woodruff-Pak et al. 1985; Solomon et al. 1986; Moyer et al. 1990) but is less critical during memory retrieval (Kim et al. 1995). We would expect differential hippocampal activity to also be observed on day 1 as well but we do not. Finally, the left and right somatosensory cortex show a significant post hoc difference on day 10 only. Given the fact that somatosensory cortex is important for all stages of tEBC (Galvez et al. 2007), we would expect this difference to be observed on day 1 and Retention day 2 but this was not seen. As a result, hippocampal and prelimbic connectivity prior to CR and non-CR trials may be different at distinct phases of the learning process, respectively. Previous studies that examined the role of the hippocampus during the pretrial period used theta activity as a surrogate of hippocampal activity and found that theta-contingent stimulus presentations accelerated tEBC acquisition (Seager et al. 2002; Griffin et al. 2004; Hoffmann and Berry 2009; Darling et al. 2011). They also found that cerebellar and hippocampal field potentials $(3.5-8.5 \mathrm{~Hz})$ were highly synchronized in time commencing with onset of the CS (Hoffmann and Berry 2009; Berry and Hoffmann 2011). This suggests that they were detecting correlations during the trial period and were able to do so due to a sampling rate that is faster than that used during collection of fMRI BOLD data (2.5-sec sampling rate). In contrast, our results are based on an analysis of pretrial activity that was not specifically analyzed by Berry and colleagues.

The greater the negative connectivity between the left SI and right IPN, the more accurate the timing of the CR is to the US onset. It does not appear that left SI $\leftrightarrow$ right IPN connectivity prior to
CR trials is related to an aspect of the behavioral response as a function of learning phase. The strength of the negative left SI $\leftrightarrow$ right IPN connectivity does not significantly differ across the three recording days. Also, the motor responses do not significantly differ across recording days. Finally, the observed correlations found between left SI $\leftrightarrow$ right IPN connectivity prior to CR trials and the duration/onset of the CR response $200 \mathrm{msec}$ prior to US onset did not significantly differ across the three recording days.

Our time delay analysis did not show improved correlation strength between the left SI and right IPN. Instead, lagging either time course by $2.5 \mathrm{sec}$ (i.e., 1TR) slightly reduced pretrial connectivity and longer lags (i.e., 5-7.5 sec) all but abolished the pretrial connectivity between the regions. FMRI analysis techniques that attempt to determine whether one temporal profile predicts the other (Stephan and Roebroeck 2012) are able to detect neuronal delays on the order of hundreds of milliseconds (Deshpande et al. 2010). However, responses in the IPN are elicited $\sim 10-40$ msec after somatosensory stimulation (Allen et al. 1977; Armstrong and Rawson 1979; Cody et al. 1981). Given the temporal resolution of our functional MRI data sets $(2.5 \mathrm{sec})$, it is not surprising that the connectivity between the two regions does not get stronger when a lag is introduced. Future studies using a shorter TR or electrophysiological studies that measure neuronal responses on the millisecond level may show the effects of lagging the time series of the SI and IPN.

Establishing the difference in network strength prior to CR versus non-CR trials leads to a correlative, but not causative, result. To establish a causal link between the network state and CR facilitation, real-time brain activity can be rapidly analyzed to deliver tEBC trials during the optimal state of negative left SI $\leftrightarrow$ right IPN connectivity which might facilitate the production of CRs, improve learning rates, and potentiate additional networks necessary for learning (i.e., prefrontal, hippocampal, etc.). This technique has been used previously in human subjects to trigger visual scenes based on real-time parahippocampal activity resulting in enhanced recognition memory during a subsequent test (Yoo et al. 2012).

The ability to prime or alter pretrial networks opens avenues that may accelerate learning and strengthen memory on associative tasks. Providing online feedback of brain activity has been shown to enable modulation of connectivity in attention-related regions (Lee et al. 2012) as well as subcortical and supplementary motor area networks (Hampson et al. 2011). Using transcranial magnetic stimulation (TMS) to excite the frontopolar cortex improves confidence ratings of memory encoding (Ryals et al. 2015) and functional connectivity changes have been observed in hippocampal networks after TMS of the lateral parietal cortex which has high functional connectivity with the hippocampus (Wang et al. 2014; Wang and Voss 2015).

This study reveals that the left SI $\leftrightarrow$ right IPN shows negative connectivity prior to $\mathrm{CR}$ trials versus non-CR trials suggesting that this circuit plays a role in efficient neural communication across regions essential for learning and performing a difficult, associative task (i.e., whisker-signaled tEBC on the right side of the rabbit). Further testing of optimal brain states prior to stimulus presentations in a causative manner may be used to enhance performance and improve the way information is acquired and retained.

\section{Materials and Methods}

\section{Subjects and surgery}

Nine female, New Zealand White rabbits $(2-4 \mathrm{~kg})$ were used in the current study. Surgery was performed under NIH and Northwestern University IACUC approved protocols to implant a restraining 
bolt assembly onto the rabbit's skull to fix the head in our custombuilt MR cradle. Anesthesia was induced in an induction box with 3\%-4\% isoflurane and maintained with $2 \%-3 \%$ isoflurane mixed with $2 \mathrm{~L} / \mathrm{min} \mathrm{O}_{2}$. Buprenex $(0.03 \mathrm{mg} / \mathrm{kg}$, s.c.) was administered to minimize discomfort during and after the procedure and ophthalmic ointment was applied to keep the eyes moist. After rabbits were placed into a stereotaxic apparatus, the shaved and cleaned scalp was incised and the skull was positioned with lambda $1.5 \mathrm{~mm}$ below bregma. In order to secure the headpost onto the skull in the stereotaxic plane (Sawyer et al. 1954; Girgis and Shih-Chang 1981), six holes (four rostral to bregma and two lateral to lambda) were drilled into (but not through) the skull. Nylon machine screws were turned into holes threaded with a $2-56$ bottoming tap. MR-compatible, nonartifact inducing Grip cement (Dentsply) was then placed on the skull and machine screws, and a custom-built headpost assembly (four upright nylon bolts $(6-32 \times 3 / 4$ in)) encased in Grip cement) was lowered onto the cement-covered skull. Additional cement was added as necessary to secure the headbolt assembly and cover the skull. Metacam $(0.2 \mathrm{mg} / \mathrm{kg}$, s.c. $)$ was administered once the rabbits were sternal and again $24 \mathrm{~h}$ later to provide analgesia.

\section{Animal restraint for $\mathrm{fMRI}$}

After 1 wk of post-operative recovery to insure that eating, drinking, and activity returned to normal, rabbits underwent a 1-d habituation protocol to the MRI scanner environment. For habituation and all subsequent scanning, earplugs were inserted and rabbits were placed in a prone position inside a cotton wrap and a canvas bag (Lomir) secured with Velcro. A single-channel, receive-only RF surface coil was secured to the underside of a Plexiglas crossbar and secured onto the rabbit's headpost with four nylon nuts. The crossbar was fastened to the custom-built cradle to stabilize the rabbit's head in the stereotaxic plane and prevent movement. The cradle, with the headposted rabbit fixed inside, was placed in the MR scanner and a $1 \mathrm{~h}$ echo-planar imaging (EPI) sequence was performed to fulfill habituation training.

Criterion for MR habituation was achieved after a single session, i.e., no signs of excessive movement (i.e., $>0.3 \mathrm{~mm}$ ) lasting longer than 2 TRs (i.e., $5 \mathrm{sec}$ ) occurred during visualization of EPI images in real-time. Although we did not collect any measurement to ascertain the stress of the rabbit (e.g., corticosterone levels), rabbits did not display any signs of overt stress (i.e., struggling) and direct monitoring of the digital waveform generated by a respiration pillow revealed consistent and maintained breathing patterns not interrupted by any excessive movement throughout the duration of habituation scanning.

\section{Experimental task design}

Rabbits underwent 60 trials of $\mathrm{tEBC}$ for each of ten consecutive days, of which the first and tenth day of conditioning was performed inside the MR scanner (Fig. 4). The second through

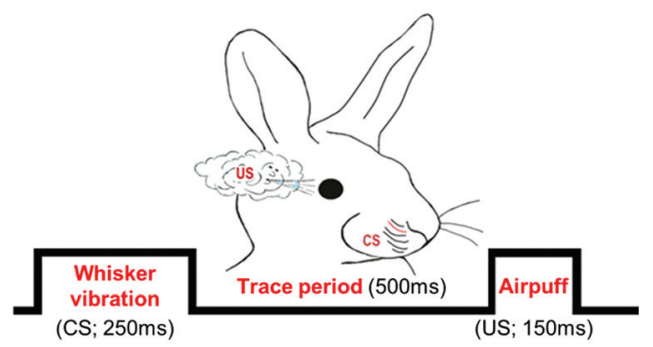

Figure 4. Experimental paradigm for $\mathrm{tEBC}$. Rabbits received 60 trials like that shown above; 250 msec conditioned stimulus (CS; whisker stimulation) followed by a $500 \mathrm{msec}$, stimulus-free trace interval and then a $150-\mathrm{msec}$ unconditioned stimulus (US; corneal airpuff). Each trial is followed by an average intertrial interval of $45 \mathrm{sec}$ (range $=30-60 \mathrm{sec}$ ). ninth days of conditioning were performed in a light and sound attenuating chamber (Med-Associates, Inc.). In order to minimize any contextual differences between the two training environments, rabbits were prepared in a similar manner and MR gradient noise $(\sim 80 \mathrm{~dB})$ played in the background of the chambers to mimic the environmental stimuli of the MRI. Following the tenth day of conditioning, rabbits remained in their home cage for $30 \mathrm{~d}$. Rabbits then underwent $2 \mathrm{~d}$ of tEBC to assess retention, the second of which was performed in the MR scanner.

tEBC trials consisted of a $250 \mathrm{msec}$ conditioned stimulus (CS; whisker stimulation) followed by a $500 \mathrm{msec}$, stimulus-free trace interval and a $150 \mathrm{msec}$ unconditioned stimulus (US; corneal airpuff). An intertrial interval (ITI; average $=45 \mathrm{sec}$; range: $30-60 \mathrm{sec}$ ) followed each trial. Extension of the nictitating membrane within the $20 \mathrm{msec}$ prior to US onset (operationalized as $\mathrm{a} \geq 15 \mathrm{msec}$ voltage increase 4 SDs above the mean of a 250-msec pre-CS baseline amplitude) was considered an adaptive conditioned response (CR). Extension of the nictitating membrane that met the above criteria, but which was not present within $20 \mathrm{msec}$ prior to US onset, was considered a nonadaptive conditioned response. Failure to extend the nictitating membrane until after US onset or an extension not satisfying the above criteria (i.e., a voltage increase $\leq 15 \mathrm{msec}$ or $<4$ SDs above the mean baseline amplitude) was considered a non-CR; (Fig. 5A).

For $\mathrm{tEBC}$ training, row $\mathrm{B}$ whiskers on the right side of the face were held within a thin strip of Post-It note (Das et al. 2001) and attached to a vibrotactile transducer (Piezo Systems) generating $\sim 100 \mu \mathrm{m}$ dorsal-ventral deflections at $60 \mathrm{~Hz}$ for CS delivery. A regulator and solenoid valve using a system described previously (Li et al. 2003) delivered 3 psi of compressed air through a small plastic tube directed at the cornea (US). To measure extension of the nictitating membrane across the cornea, the right eyelid was held open with tailor hooks and a fiber optic cable assembly transmitting infrared light to and from an infrared emitter and detector was positioned $\sim 1 \mathrm{~cm}$ in front of the right eye to measure the change in voltage (Miller et al. 2005). Eyeblink data were sampled at $1 \mathrm{kHz}$. A computer running custom Labview software (National Instruments, Inc.) controlled stimulus delivery and behavioral data collection.

\section{MRI data acquisition}

MR scanning was conducted in a Bruker $7 \mathrm{~T} / 30$-cm wide horizontal magnet (ClinScan, Bruker Biospin) using a single-channel, receive-only RF surface coil with an inner diameter of $30 \mathrm{~mm}$. Transmission was achieved with a two channel volume coil fixed inside the magnet. Repositioning of the same animal was achieved in all three directions $(X, Y$, and $Z)$ with great accuracy $(<500 \mu \mathrm{m})$ across sessions. Adjustments to optimize shimming, reduce air-tissue artifacts and produce a uniform magnetic field were performed on a manually selected region (centered on, but not exceeding the size of, the rabbit brain). First and second order shimming was performed using an automated field map algorithm included in the Syngo platform. Shim tables showing the resulting $x, y, z$ and higher order shim values confirmed no major variability in shim values across subjects and sessions. A highresolution $\mathrm{T}_{1}$-weighted anatomical reference was first acquired (1.0-mm slice thickness, $0.5 \times 0.5-\mathrm{mm}$ in-plane resolution, FOV $=64 \times 64 \mathrm{~mm}$, matrix size $=128 \times 128 \times 40$, repetition time $(\mathrm{TR})=600 \mathrm{msec}$, echo time $(\mathrm{TE})=2.09 \mathrm{msec}$, flip angle $=$ $45^{\circ}, 40$ slices). Blood-oxygen-level dependent (BOLD) contrastsensitive $\mathrm{T}_{2}{ }^{*}$-weighted gradient-echo echo-planar images (EPI) covering the entire rabbit brain were acquired during trace eyeblink conditioning $(2.0 \mathrm{~mm}$ slice thickness, $0.5 \times 0.5-\mathrm{mm}$ in-plane resolution, $\mathrm{FOV}=35 \times 26 \mathrm{~mm}$; matrix size $=70 \times$ $52 \times 20, \mathrm{TR}=2.5 \mathrm{sec}, \mathrm{TE}=25 \mathrm{msec}$, flip angle $=90^{\circ}, 20$ coronal slices, 1080 volumes).

\section{BOLD EPI processing and data analysis}

Data analysis was performed with AFNI (Cox 1996). The first four volumes of each functional data set were discarded to 

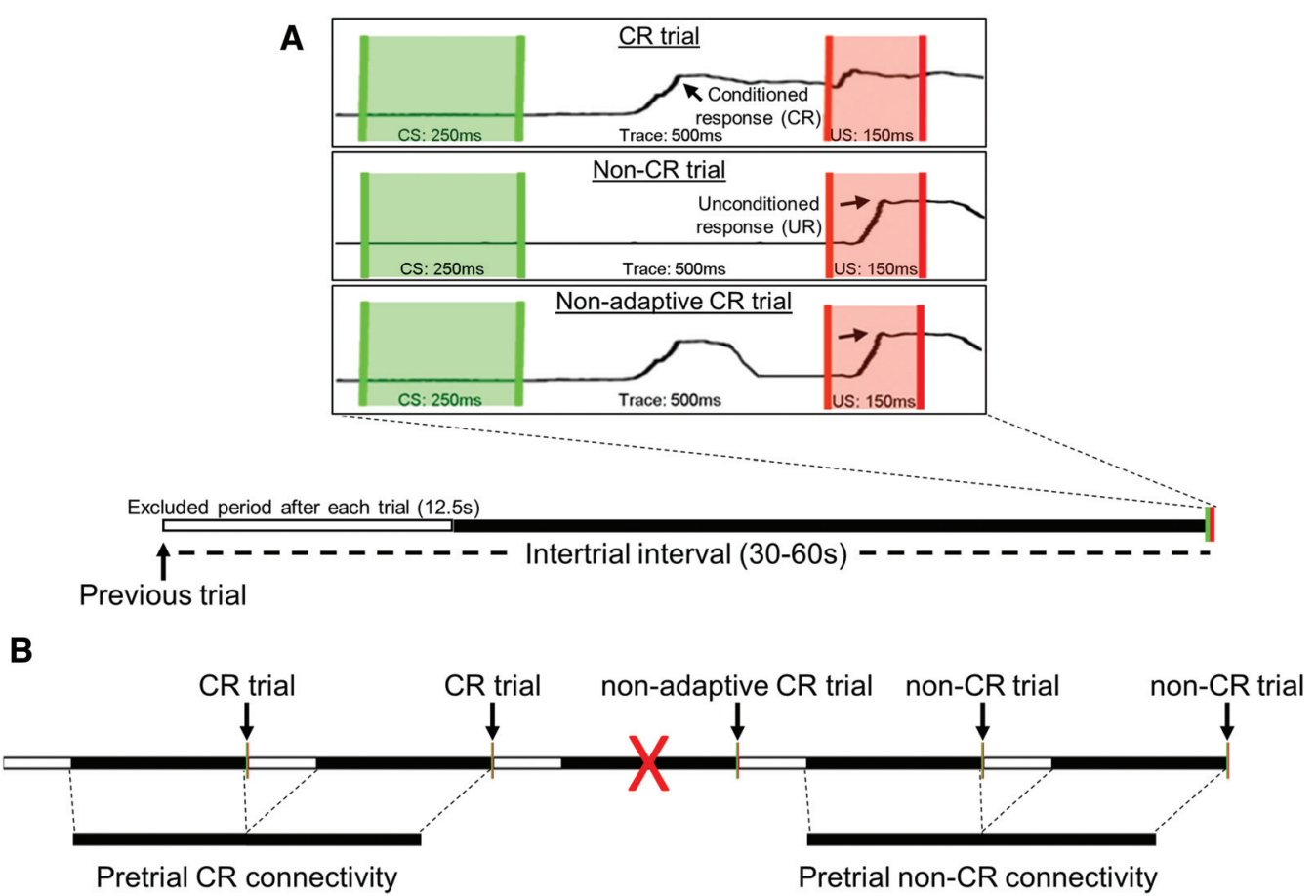

Figure 5. Illustration of intertrial interval and example $C R$ and non-CR trials used to characterize pretrial connectivity. $(A)$ Adaptive $C R$ trials are characterized by a change in voltage amplitude caused by extension of the nictitating membrane in anticipation of the unconditioned stimulus (US; i.e., airpuff), producing a conditioned response that is present within $20 \mathrm{msec}$ of US onset. Non-CR trials are characterized by the absence of a voltage change until after US presentation producing an unconditioned response. Non-adaptive CR trials are characterized by a change in voltage prior to US presentation but a return to baseline within $20 \mathrm{msec}$ of US onset. After each trial, an intertrial interval occurred (mean $=45 \mathrm{sec}$; range 30-60 sec). The first $12.5 \mathrm{sec}$ after each trial was excluded from pretrial connectivity analysis. (B) Illustration of five hypothetically consecutive trials and parsing of pretrial CR and non-CR connectivity. Note, pretrial connectivity for nonadaptive CR trials was not analyzed as denoted by the red X.

account for eddy currents and NMR equilibrium. After performing slice-timing and motion correction on each EPI data set, displacement of each brain volume relative to the previous volume was calculated as the Euclidian norm of the translational $(x, y, z)$ and rotational $(\alpha, \beta, \gamma)$ rigid-body motion correction parameters (displacement $=$ square root of $\left[(\Delta x)^{2}+(\Delta y)^{2}+(\Delta z)^{2}+\right.$ $\left.(\Delta \alpha)^{2}+(\Delta \beta)^{2}+(\Delta \gamma)^{2}\right]$ ) (Belcher et al. 2013). Since rotational or translational displacement did not exceed our criterion of $0.3 \mathrm{~mm}$, no data points were eliminated due to excessive motion. The average maximum displacement across the entire subject population was $0.09 \mathrm{~mm}$ (s.d. $=0.04 \mathrm{~mm}$ ). EPI images from each rabbit were coaligned with the $\mathrm{T}_{1}$-weighted anatomical reference scan collected during the same session. $\mathrm{T}_{1}$-weighted anatomical scans were then spatially aligned to a separate, previously collected, high-resolution rabbit brain $\left(0.2 \mathrm{~mm}^{3}\right.$ resolution) and the same transformation was applied to the EPI images. EPI data sets were then spatially smoothed $(\mathrm{FWHM}=0.71 \mathrm{~mm})$.

In order to characterize pretrial connectivity without confounds of task-induced activation, a general linear model was used to model each 0.9-sec tEBC trial with a boxcar hemodynamic response function and its temporal derivative. Additionally, the six motion parameters $(x, y, z$ shifts and yaw, pitch, roll rotations) were included as regressors of no interest. The resulting residual time series (produced after modeling task-related activation and regressing out motion parameters) was saved and temporally filtered $(0.005-0.1 \mathrm{~Hz})$ to exclude low- and highfrequency fluctuations. Data during the five TRs after the end of each trial were discarded and not included in the analysis to avoid potential contamination with BOLD signals not regressed out during the first-level analysis (Fig. 5A). Residual time series were then parcellated and concatenated (Fair et al. 2007) depending on whether a CR or non-CR occurred on the upcoming trial (Fig. 5B). Because the percentage of those nonadaptive CR trials was low across sessions (e.g., day 1: 12.1\%, day 10:
$17.7 \%$, Retention day 2: $27.3 \%$ ), analysis of this trial type was underpowered and required that upcoming trials in which a nonadaptive CR occurred be discarded from the analysis. The average number of concatenated time points per upcoming trial type (i.e., CR and non-CR) across scan sessions was 423 (s.d.= 229; range: 100-924).

Regions of interest in the left and right hemispheres were drawn in the cerebellar interpositus nucleus (IPN), primary somatosensory cortex (SI), hippocampus and prelimbic cortices. Pearson correlations were separately calculated between each hemispheric and regional combination for concatenated ITI periods prior to CR or non-CR trials (Fig. 5B). In order to perform group analysis, correlations (which range from -1 to +1 ) underwent Fisher's Z-transformation to reduce skewness and make the sampling distribution more normal (a prerequisite of the statistical methods used). Z-transformed correlations were subjected to a linear mixed model with factors for day (day 1, day 10, and retention day 2) and upcoming trial type (CR versus non-CR on the upcoming trials) with a significance level of $P \leq 0.05$ (corrected for multiple regional comparisons).

\section{Time delay analysis}

A time delay analysis was performed to determine whether connectivity between the regional combinations became more robust when time courses were lagged relative to each other. The previously parcellated and concatenated residual time series were lagged 2.5, 5, and $7.5 \mathrm{sec}$ (i.e., 1, 2, and 3 TRs) relative to the other time series. Intertrial interval time points that no longer had a corresponding time point with the other times series were discarded. Pearson correlations were separately calculated between each hemispheric and regional combination for concatenated ITI periods prior to CR or non-CR trials. Correlations were determined as previously mentioned. 


\section{Acknowledgments}

This work was supported by the National Institutes of Health (National Institute of Neurological Disorders and Stroke Grant no. R01NS059879 to C.W. and the National Institute of Mental Health Grant no. R01 MH047340 to J.F.D.). M.P.S. was supported by the T32 Training Program: The Neuroscience of Human Cognition at Northwestern University (T32-NS047987).

\section{Competing interest statement}

The authors declare no competing financial interests.

\section{References}

Allen GI, Gilbert PF, Marini R, Schultz W, Yin TC. 1977. Integration of cerebral and peripheral inputs by interpositus neurons in monkey. Exp Brain Res 27: 81-99.

Armstrong DM, Rawson JA. 1979. Responses of neurones in nucleus interpositus of the cerebellum to cutaneous nerve volleys in the awake cat. J Physiol 289: 403-423.

Belcher AM, Yen CC, Stepp H, Gu H, Lu H, Yang Y, Silva AC, Stein EA. 2013. Large-scale brain networks in the awake, truly resting marmoset monkey. J Neurosci 33: 16796-16804.

Berry SD, Hoffmann LC. 2011. Hippocampal theta-dependent eyeblink classical conditioning: coordination of a distributed learning system. Neurobiol Learn Mem 95: 185-189.

Berry SD, Thompson RF. 1978. Prediction of learning rate from the hippocampal electroencephalogram. Science 200: 1298-1300.

Brodal A. 1981. Neurological anatomy in relation to clinical medicine. Oxford University, New York.

Christian KM, Thompson RF. 2003. Neural substrates of eyeblink conditioning: acquisition and retention. Learn Mem 10: 427-455.

Christian KM, Thompson RF. 2005. Long-term storage of an associative memory trace in the cerebellum. Behav Neurosci 119: 526-537.

Chun MM, Turk-Browne NB. 2007. Interactions between attention and memory. Curr Opin Neurobiol 17: 177-184.

Clark GA, McCormick DA, Lavond DG, Thompson RF. 1984. Effects of lesions of cerebellar nuclei on conditioned behavioral and hippocampal neuronal responses. Brain Res 291: 125-136.

Cody FW, Moore RB, Richardson HC. 1981. Patterns of activity evoked in cerebellar interpositus nuclear neurones by natural somatosensory stimuli in awake cats. J Physiol 317: 1-20.

Cohen N, Pell L, Edelson MG, Ben-Yakov A, Pine A, Dudai Y. 2015. Peri-encoding predictors of memory encoding and consolidation. Neurosci Biobehav Rev 50: 128-142.

Cox RW. 1996. AFNI: software for analysis and visualization of functional magnetic resonance neuroimages. Comput Biomed Res 29: 162-173.

Darling RD, Takatsuki K, Griffin AL, Berry SD. 2011. Eyeblink conditioning contingent on hippocampal theta enhances hippocampal and medial prefrontal responses. J Neurophysiol 105: 2213-2224.

Das S, Weiss C, Disterhoft JF. 2001. Eyeblink conditioning in the rabbit (Oryctolagus cuniculus) with stimulation of the mystacial vibrissae as a conditioned stimulus. Behav Neurosci 115: 731-736.

Deshpande G, Sathian K, Hu X. 2010. Effect of hemodynamic variability on Granger causality analysis of fMRI. Neuroimage 52: 884-896.

Driver J, Frith C. 2000. Shifting baselines in attention research. Nat Rev Neurosci 1: $147-148$.

Fair DA, Schlaggar BL, Cohen AL, Miezin FM, Dosenbach NUF, Wenger KK, Fox MD, Snyder AZ, Raichle ME, Petersen SE. 2007. A method for using blocked and event-related fMRI data to study "resting state" functional connectivity. Neuroimage 35: 396-405.

Galvez R, Weible AP, Disterhoft JF. 2007. Cortical barrel lesions impair whisker-CS trace eyeblink conditioning. Learn Mem 14: 94-100.

Girgis M, Shih-Chang W. 1981. A new stereotaxic atlas of the rabbit brain. Warren H. Green, Inc., St. Louis, MO.

Glickstein M, Cohen JL, Dixon B, Gibson A, Hollins M, Labossiere E, Robinson F. 1980. Corticopontine visual projections in macaque monkeys. J Comp Neurol 190: 209-229.

Green JT, Arenos JD. 2007. Hippocampal and cerebellar single-unit activity during delay and trace eyeblink conditioning in the rat. Neurobiol Learn Mem 87: 269-284.

Griffin AL, Asaka Y, Darling RD, Berry SD. 2004. Theta-contingent trial presentation accelerates learning rate and enhances hippocampal plasticity during trace eyeblink conditioning. Behav Neurosci 118: $403-411$.

Hampson M, Scheinost D, Qiu M, Bhawnani J, Lacadie CM, Leckman JF, Constable RT, Papademetris X. 2011. Biofeedback of real-time functional magnetic resonance imaging data from the supplementary motor area reduces functional connectivity to subcortical regions. Brain Connect 1: 91-98.

Hattori S, Yoon T, Disterhoft JF, Weiss C. 2014. Functional reorganization of a prefrontal cortical network mediating consolidation of trace eyeblink conditioning. J Neurosci 34: 1432-1445.

Hoffmann LC, Berry SD. 2009. Cerebellar theta oscillations are synchronized during hippocampal theta-contingent trace conditioning. Proc Natl Acad Sci 106: 21371-21376.

Hu B, Chen H, Feng H, Zeng Y, Yang L, Fan ZL, Wu YM, Sui JF. 2010. Disrupted topography of the acquired trace-conditioned eyeblink responses in guinea pigs after suppression of cerebellar cortical inhibition to the interpositus nucleus. Brain Res 1337: 41-55.

Hu B, Chen H, Yang L, Tao ZF, Yan J, Zhang YH, Zhu ZR, Sun WZ, Huang W, Huang WQ et al. 2012. Changes of synaptic ultrastructure in the guinea pig interpositus nuclei associate with response magnitude and timing after trace eyeblink conditioning. Behav Brain Res 226: 529-537.

Kim JJ, Clark RE, Thompson RF. 1995. Hippocampectomy impairs the memory of recently, but not remotely, acquired trace eyeblink conditioned responses. Behav Neurosci 109: 195-203.

König P, Engel AK, Roelfsema PR, Singer W. 1995. How precise is neuronal synchronization? Neural Comput 7: 469-485.

Kronforst-Collins MA, Disterhoft JF. 1998. Lesions of the caudal area of rabbit medial prefrontal cortex impair trace eyeblink conditioning. Neurobiol Learn Mem 69: 147-162.

Krupa DJ, Thompson RF. 1995. Inactivation of the superior cerebellar peduncle blocks expression but not acquisition of the rabbit's classically conditioned eye-blink response. Proc Natl Acad Sci 92: 5097-5101.

Krupa DJ, Thompson RF. 1997. Reversible inactivation of the cerebellar interpositus nucleus completely prevents acquisition of the classically conditioned eye-blink response. Learn Mem 3: 545-556.

Krupa DJ, Thompson JK, Thompson RF. 1993. Localization of a memory trace in the mammalian brain. Science 260: 989-991.

Krupa DJ, Weng J, Thompson RF. 1996. Inactivation of brainstem motor nuclei blocks expression but not acquisition of the rabbit's classically conditioned eyeblink response. Behav Neurosci 110: 219-227.

Lavond DG, Steinmetz JE. 1989. Acquisition of classical conditioning without cerebellar cortex. Behav Brain Res 33: 113-164.

Lee J-H, Kim J, Yoo S-S. 2012. Real-time fMRI-based neurofeedback reinforces causality of attention networks. Neurosci Res 72: 347-354.

Li L, Weiss C, Disterhoft JF, Wyrwicz AM. 2003. Functional magnetic resonance imaging in the awake rabbit: a system for stimulus presentation and response detection during eyeblink conditioning. J Neurosci Methods 130: 45-52.

Ma WJ, Beck JM, Latham PE, Pouget A. 2006. Bayesian inference with probabilistic population codes. Nat Neurosci 9: 1432-1438.

McCormick DA, Clark GA, Lavond DG, Thompson RF. 1982. Initial localization of the memory trace for a basic form of learning. Proc Natl Acad Sci 79: 2731-2735.

McLaughlin J, Powell DA, White JD. 2002. Characterization of the neuronal changes in the medial prefrontal cortex during jaw movement and eyeblink Pavlovian conditioning in the rabbit. Behav Brain Res 132: $117-133$.

Medina JF, Repa JC, Mauk MD, LeDoux JE. 2002. Parallels between cerebellum- and amygdala-dependent conditioning. Nat Rev Neurosci 3: $122-131$.

Miller EK, Cohen JD. 2001. An integrative theory of prefrontal cortex function. Annu Rev Neurosci 24: 167-202.

Miller MJ, Chen NK, Li L, Tom B, Weiss C, Disterhoft JF, Wyrwicz AM. 2003. fMRI of the conscious rabbit during unilateral classical eyeblink conditioning reveals bilateral cerebellar activation. J Neurosci 23: 11753-11758.

Miller MJ, Li L, Weiss C, Disterhoft JF, Wyrwicz AM. 2005. A fiber optic-based system for behavioral eyeblink measurement in a MRI environment. J Neurosci Methods 141: 83-87.

Moyer JR Jr, Deyo RA, Disterhoft JF. 1990. Hippocampectomy disrupts trace eye-blink conditioning in rabbits. Behav Neurosci 104: 243-252.

Nikolić D. 2007. Non-parametric detection of temporal order across pairwise measurements of time delays. J Comput Neurosci 22: 5-19.

Noudoost B, Chang MH, Steinmetz NA, Moore T. 2010. Top-down control of visual attention. Curr Opin Neurobiol 20: 183-190.

Pakaprot N, Kim S, Thompson RF. 2009. The role of the cerebellar interpositus nucleus in short and long term memory for trace eyeblink conditioning. Behav Neurosci 123: 54-61.

Plakke B, Freeman JH, Poremba A. 2007. Metabolic mapping of the rat cerebellum during delay and trace eyeblink conditioning. Neurobiol Learn Mem 88: 11-18.

Ryals AJ, Rogers LM, Gross EZ, Polnaszek KL, Voss JL. 2015. Associative recognition memory awareness improved by theta-burst stimulation of frontopolar cortex. Cereb Cortex doi: 10.1093/cercor/bhu311.

Sawyer CH, Everett JW, Green JD. 1954. The rabbit diencephalon in stereotaxic coordinates. J Comp Neurol 101: 801-824. 
Schmahmann JD, Pandya DN. 1989. Anatomical investigation of projections to the basis pontis from posterior parietal association cortices in rhesus monkey. J Comp Neurol 289: 53-73.

Schmahmann JD, Pandya DN. 1991. Projections to the basis pontis from the superior temporal sulcus and superior temporal region in the rhesus monkey. J Comp Neurol 308: 224-248.

Schmahmann JD, Pandya DN. 1993. Prelunate, occipitotemporal, and parahippocampal projections to the basis pontis in rhesus monkey. J Comp Neurol 337: 94-112.

Schneider G, Havenith MN, Nikolić D. 2006. Spatiotemporal structure in large neuronal networks detected from cross-correlation. Neural Comput 18: 2387-2413.

Seager MA, Johnson LD, Chabot ES, Asaka Y, Berry SD. 2002. Oscillatory brain states and learning: impact of hippocampal theta-contingent training. Proc Natl Acad Sci 99: 1616-1620.

Solomon PR, Vander Schaaf ER, Thompson RF, Weisz DJ. 1986. Hippocampus and trace conditioning of the rabbit's classically conditioned nictitating membrane response. Behav Neurosci 100: $729-744$.

Stephan KE, Roebroeck A. 2012. A short history of causal modeling of fMRI data. Neuroimage 62: 856-863.

Takehara K, Kawahara S, Kirino Y. 2003. Time-dependent reorganization of the brain components underlying memory retention in trace eyeblink conditioning. J Neurosci 23: 9897-9905.

van den Berg R, Shin H, Chou W-C, George R, Ma WJ. 2012. Variability in encoding precision accounts for visual short-term memory limitations. Proc Natl Acad Sci 109: 8780-8785.

Wang JX, Voss JL. 2015. Long-lasting enhancements of memory and hippocampal-cortical functional connectivity following multiple-day targeted noninvasive stimulation. Hippocampus 25: 877-883.

Wang JX, Rogers LM, Gross EZ, Ryals AJ, Dokucu ME, Brandstatt KL, Hermiller MS, Voss JL. 2014. Targeted enhancement of cortical-hippocampal brain networks and associative memory. Science 345: $1054-1057$.

Ward RL, Flores LC, Disterhoft JF. 2012. Infragranular barrel cortex activity is enhanced with learning. J Neurophysiol 108: 1278-1287.

Weible AP, McEchron MD, Disterhoft JF. 2000. Cortical involvement in acquisition and extinction of trace eyeblink conditioning. Behav Neurosci 114: 1058-1067.

Weiss C, Bouwmeester H, Power JM, Disterhoft JF. 1999. Hippocampal lesions prevent trace eyeblink conditioning in the freely moving rat. Behav Brain Res 99: 123-132.

Wikgren J, Ruusuvirta T, Korhonen T. 2003. Activity in the rabbit somatosensory cortex reflects the active procedural memory trace of a classically conditioned eyeblink response. Neurosci Lett 341: 119-122.

Woodruff-Pak DS, Disterhoft JF. 2008. Where is the trace in trace conditioning? Trends Neurosci 31: 105-112.

Woodruff-Pak DS, Lavond DG, Thompson RF. 1985. Trace conditioning: abolished by cerebellar nuclear lesions but not lateral cerebellar cortex aspirations. Brain Res 348: 249-260.

Wu GY, Yao J, Fan ZL, Zhang LQ, Li X, Zhao CD, Zhou ZH, Sui JF. 2012. Classical eyeblink conditioning using electrical stimulation of caudal mPFC as conditioned stimulus is dependent on cerebellar interpositus nucleus in guinea pigs. Acta Pharmacol Sin 33: 717-727.

Yeo CH, Hardiman MJ, Glickstein M. 1985. Classical conditioning of the nictitating membrane response of the rabbit. I. Lesions of the cerebellar nuclei. Exp Brain Res 60: 87-98.

Yoo JJ, Hinds O, Ofen N, Thompson TW, Whitfield-Gabrieli S, Triantafyllou C, Gabrieli JDE. 2012. When the brain is prepared to learn: enhancing human learning using real-time fMRI. Neuroimage 59: $846-852$.

Received September 20, 2015; accepted in revised form January 21, 2016. 


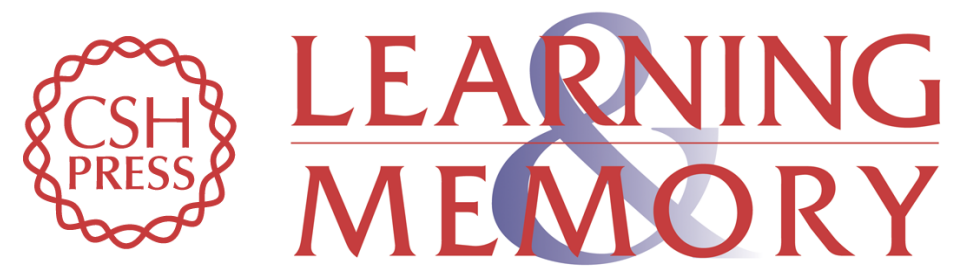

\section{Pretrial functional connectivity differentiates behavioral outcomes during trace eyeblink conditioning in the rabbit}

Matthew P. Schroeder, Craig Weiss, Daniel Procissi, et al.

Learn. Mem. 2016, 23:

Access the most recent version at doi:10.1101/Im.040220.115

\section{Supplemental http://learnmem.cshlp.org/content/suppl/2016/03/10/23.4.161.DC1 Material}

References This article cites 70 articles, 15 of which can be accessed free at: http://learnmem.cshlp.org/content/23/4/161.full.html\#ref-list-1

Creative This article is distributed exclusively by Cold Spring Harbor Laboratory Press for the Commons first 12 months after the full-issue publication date (see

License http://learnmem.cshlp.org/site/misc/terms.xhtml). After 12 months, it is available under a Creative Commons License (Attribution-NonCommercial 4.0 International), as described at http://creativecommons.org/licenses/by-nc/4.0/.

Email Alerting Receive free email alerts when new articles cite this article - sign up in the box at the Service top right corner of the article or click here. 